\title{
X-Ray Measurements of Noncapillary Spatial Fluctuations from a Liquid Surface
}

\author{
M. Fukuto, ${ }^{1}$ R. K. Heilmann, ${ }^{1}$ P. S. Pershan, ${ }^{1}$ J. A. Griffiths,${ }^{2}$ S. M. Yu, ${ }^{2}$ and D. A. Tirrell ${ }^{2}$ \\ ${ }^{1}$ Department of Physics and Division of Engineering and Applied Sciences, Harvard University, Cambridge, Massachusetts 02138 \\ ${ }^{2}$ Polymer Science and Engineering Department, University of Massachusetts, Amherst, Massachusetts 01003
}

(Received 2 March 1998)

\begin{abstract}
Off-specular diffuse x-ray scattering measurements on both pure water and a homogeneous Langmuir monolayer of poly- $\gamma$-benzyl- $L$-glutamate (PBLG) on water establish the validity of a proposed sum rule for scattering from capillary fluctuations on liquid surfaces. Excess scattering above the predicted capillary contribution is observed when the PBLG monolayer is compressed beyond its elastic limit. This is interpreted in terms of a second-layer inhomogeneity with a surface correlation length of $\sim 1000 \AA$. Excess off-specular scattering can be used to probe interface correlation lengths from $100 \AA$ to $1 \mu \mathrm{m}$. [S0031-9007(98)07353-0]
\end{abstract}

PACS numbers: 68.10.-m, 61.10.Eq, 61.10.Kw, 68.35.Ct

The availability of lasers in the 1960s facilitated the first concerted application of low-angle diffuse scattering to the study of critical fluctuations in bulk condensed matter $[1,2]$. Later development of high brilliance $x$-ray sources extended these studies to shorter lengths and other materials that cannot be probed with visible light $[3,4]$. With increasing appreciation for the effects of reduced dimensions in statistical physics and with refined instrumentation, $\mathrm{x}$-ray and neutron scattering were widely applied to study two-dimensional (2D) critical properties of solid surfaces [5-7]. However, it is known that the periodic potential of the solid substrate suppresses certain classes of continuous 2D phase transitions [8,9]. Consequently attention was directed to the study of similar phenomena on liquid surfaces.

Two x-ray scattering techniques have served as useful probes for fluctuations on liquid surfaces. First, grazing incidence diffraction has been used to characterize surface structures on intermolecular length scales. The second technique, analogous to the static critical light scattering methods, is diffuse scattering at small angles away from specular reflection [10-12]. Until recently, quantitative application of this technique to liquid surfaces [13-20] has been mostly limited to characterization of thermal diffuse scattering (TDS) [11], which arises from 2D surface height fluctuations due to thermally excited capillary waves [2123]. As Sinha et al. have shown [10,14], the TDS from liquid surfaces diverges algebraically at the angle for specular reflection with strong tails extending out into the surrounding off-specular regions. We show here that calculation of the angular dependence of TDS for homogeneous liquid surfaces allows one to quantitatively separate TDS from subtle diffuse scattering effects arising from other surface inhomogeneities. This ability is essential to the utility of small-angle off-specular diffuse $\mathrm{x}$-ray scattering (OSDX) for quantitative characterization of noncapillary surface inhomogeneities [24].

We first demonstrate the application of a sum rule that leads to a simple normalized form of the capillary-wave TDS cross section predicted by Sinha et al. $[10,14]$. With this sum rule, the intensity of the OSDX can be calculated with no additional adjustable parameters beyond those required for $x$-ray specular reflectivity (XR). We apply the result to both pure $\mathrm{H}_{2} \mathrm{O}$ and a close-packed homogeneous Langmuir monolayer (LM) formed by poly- $\gamma-$ benzyl- $L$-glutamate (PBLG) molecules on water. When the PBLG film is compressed beyond the elastic limit for the monolayer, we find excess scattering above the capillary contribution that can be quantitatively interpreted in terms of surface inhomogeneities with a correlation length of $\sim 1000 \AA$.

The scattering kinematics are illustrated in Fig. 1. For $\mathrm{x}$ rays incident at angle $\alpha$ and scattered at angles $\beta$ to the surface and $2 \theta$ to the incident plane, the wave vector transfer has components $q_{z}=(2 \pi / \lambda)[\sin (\alpha)+\sin (\beta)]$ and $q_{x y}=(2 \pi / \lambda)\left[\cos ^{2}(\alpha)+\cos ^{2}(\beta)-2 \cos (2 \theta) \times\right.$ $\cos (\alpha) \cos (\beta)]^{1 / 2}$, normal and parallel to the surface. Capillary waves give rise to interfacial height-height correlations of the liquid surface $g\left(r_{x y}\right)=\left\langle\left[h\left(r_{x y}\right)-h(0)\right]^{2}\right\rangle$

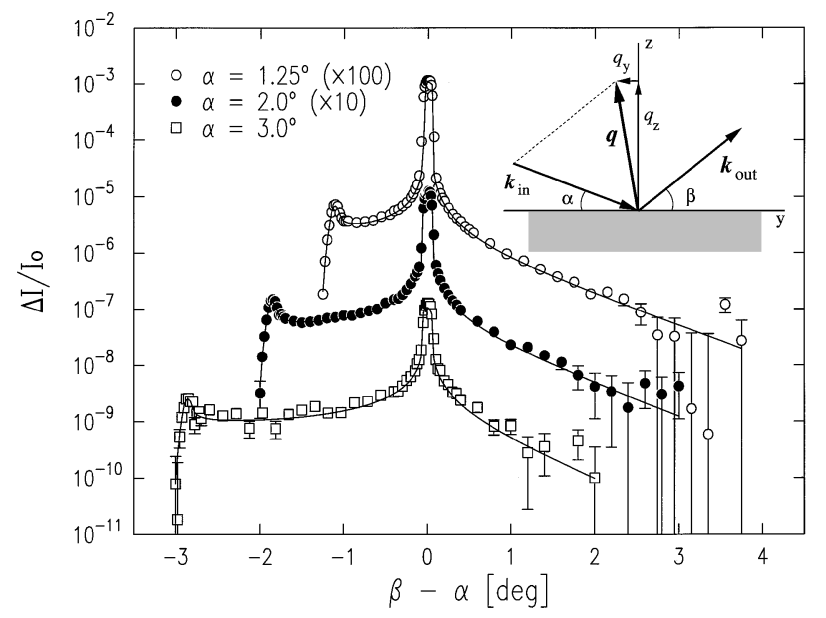

FIG. 1. Measured normalized intensity $\Delta I / I_{0}$ as a function of $\beta-\alpha$ at fixed $\alpha$ for a bare water surface. The solid lines are theoretically expected curves. The inset is a schematic for the incident-plane $(2 \theta=0)$ scattering geometry used in the XR and OSDX. 
which vary logarithmically $[10,14,23]$, when the distance $r_{x y}$ is smaller than a gravitationally imposed cutoff $(\sim \mathrm{mm})$ and large compared to the molecular size $\sim d$. Therefore, scattering from liquid surfaces does not possess a true specular reflection $\left[\sim \delta^{(2)}\left(\mathbf{q}_{x y}\right)\right]$, and the surface scattering may be considered entirely as "diffuse." Neglecting the effect of the gravitational low- $q$ cutoff (which is too small to measure with realistic resolutions), the differential cross section $d \sigma / d \Omega$ is described by a power-law singularity of the form $1 / q_{x y}^{2-\eta}$ for $\eta=\left(k_{B} T / 2 \pi \gamma\right) q_{z}^{2}<2[10,14,20]$, where $\gamma$ is the surface tension. Normalization of $d \sigma / d \Omega$ can be accomplished by taking into account the small $r_{x y}$ behavior which requires that $g(0)=0$. Since the derivation of $S\left(q_{x y}\right) \sim 1 / q_{x y}^{2-\eta}$ by Sinha et al. [10] is based on $F\left(q_{x y}\right)\left\{=2\right.$ D Fourier transform (FT) of $\exp \left[-\frac{1}{2} g\left(r_{x y}\right) \times\right.$ $\left.\left.q_{z}^{2}\right]\right\}$, it follows that the inverse FT evaluated at $r_{x y}=0$, obtained by summing $F\left(q_{x y}\right)$ over all capillary modes with $q_{x y} \leq q_{\max } \sim 2 \pi / d$, must be unity. The application of this sum rule leads to a simple and physically meaningful normalization of $S\left(q_{x y}\right)$ from Eq. (2.32) in Sinha et al. [10], without the need for a specific resolution function [14]. For the scattering from a homogeneous liquid surface (i.e., conformal roughness of all interfaces) with capillary fluctuations, one obtains [20]

$$
\frac{1}{A_{0}}\left(\frac{d \sigma}{d \Omega}\right)_{0}=\frac{N}{q_{z}^{2}}\left|\Phi_{0}\left(q_{z}\right)\right|^{2} \frac{2 \pi \eta}{q_{x y}^{2}}\left(\frac{q_{x y}}{q_{\max }}\right)^{\eta},
$$

where $A_{0}$ is the cross sectional area of the incident beam and $N=\left(q_{c} / 2\right)^{4} T_{F}(\alpha) T_{F}(\beta) /\left[16 \pi^{2} \sin (\alpha)\right]$. $q_{c}=(4 \pi / \lambda) \sin \left(\alpha_{c}\right)$ is the critical wave vector for total reflection $\left(q_{c}=0.0218 \AA^{-1}\right.$ for water) and the Fresnel transmission factor $T_{F}(\alpha)$ [10] is related to the Fresnel reflectivity $R_{F}(\alpha)$ of an ideally flat interface through $T_{F}(\alpha)=\left(2 \alpha / \alpha_{c}\right)^{2} \sqrt{R_{F}(\alpha)}$ [25]. A surface structure factor $\Phi_{0}\left(q_{z}\right)$ can be defined as the 1D FT of $d\left(\left\langle\rho_{T=0}(z)\right\rangle /\right.$ $\left.\rho_{\infty}\right) / d z$, where $\rho_{\infty}$ is the bulk electron density, and $\left\langle\rho_{T=0}(z)\right\rangle$ is the average local electron density profile in the absence of thermal capillary waves. This "intrinsic" profile $\left\langle\rho_{T=0}(z)\right\rangle[11,20,21]$ is to be distinguished from the total average density $\langle\rho(z)\rangle$, which is obtained by convoluting $\left\langle\rho_{T=0}(z)\right\rangle$ with the distribution of interfacial heights induced by capillary-wave roughness. In order to obtain the number of photons scattered into the detector normalized to the number incident on the surface (i.e., $\left.I / I_{0}\right)$, we carry out a precise numerical convolution of (1) with the slit-defined resolution function [20,26].

Details of our Langmuir trough, film preparation, and compression methods have been given previously $[27,28]$. The temperature of the pure water subphase was maintained at $23{ }^{\circ} \mathrm{C}$. The surface tension of a film-coated surface is given by $\gamma=\gamma_{\mathrm{w}}-\Pi$, where $\gamma_{\mathrm{w}}$ is for the bare water surface $(72.3 \mathrm{dyn} / \mathrm{cm})$, and $\Pi$ is the surface pressure, monitored by a Wilhelmy plate. Polydisperse PBLG [29] monolayers were spread at a specific area $A>$ $23 \AA^{2} /$ monomer from a trifluoroacetic acid/chloroform solution (3\%:97\% by volume) [30] with a concentra- tion of $0.42 \mathrm{mg} / \mathrm{mL}$ and compressed at a rate $<0.01$ $\left(\AA^{2} /\right.$ monomer $) / s$. The PBLG molecule, due to its $\alpha$-helical conformation, resembles a rodlike structure approximately $150 \AA$ in length and $13 \AA$ in diameter [30,31]. On water these rods lie down parallel to the interface [31].

$\mathrm{X}$-ray experiments were carried out using the Harvard/ BNL liquid surface spectrometer [27] on Beamline X22B at the National Synchrotron Light Source $(\lambda=1.55 \AA)$. For both XR and OSDX, the center of the detector (NaI scintillator) slits lies in the incident plane $(2 \theta=0)$. For $\mathrm{XR}$, the reflected intensity at $\beta=\alpha$ (or $q_{x y}=0$ ) is measured as a function of $q_{z}=(4 \pi / \lambda) \sin (\alpha)$, while OSDX was measured as a function of $\beta$ at fixed $\alpha$. For both measurements the background was eliminated through subtraction of intensities from identical scans taken with $2 \theta$ offsets of $\pm 0.3^{\circ}$. The results were normalized to the incident intensity and analyzed in terms of the theoretical predictions for the difference $\Delta I / I_{0} \equiv\{I(2 \theta=0)-$ $\left.(1 / 2)\left[I\left(+0.3^{\circ}\right)+I\left(-0.3^{\circ}\right)\right]\right\} / I_{0}[20]$. The rectangular detector slits, located $L=621 \mathrm{~mm}$ from the sample center, of (height $H) \times($ width $W$ ) give an angular resolution of $\delta \beta=H / L$ and $\delta(2 \theta)=W / L$. The slit sizes in $\mathrm{mm}$ were $(H, W)=(2.5,3.0)$ for $\mathrm{XR},(1.1,3.0)$ for $\beta$ scans on water, and $(1.0,3.0)$ for $\beta$ scans on PBLG films.

Figure 1 shows $\Delta I / I_{0}$ for $\beta$ scans taken on water. The local density profile $\rho_{T=0}(\mathbf{r})=\left\langle\rho_{T=0}(z)\right\rangle$ at any point on the simple water/gas interface is a step function, so that $\Phi_{0}\left(q_{z}\right)=1$ [13]. The solid lines are calculated values of $\Delta I / I_{0}$ using the known temperature, surface tension, and $q_{\max }=\pi /(1.4 \AA)=2.25 \AA^{-1}$ as obtained previously for water [13]. The calculation involves no adjustable parameters. The theory agrees excellently with the measurements for both the specular and offspecular data. Conservative estimates of uncertainties in the experimentally determined parameters $(T, \gamma, \delta \beta$, $\delta(2 \theta)$, etc.) lead to errors in the shown curves that are much smaller than the size of the symbols for the data.

The validity of the formula (1) was tested again for a less simple interfacial structure using the PBLG LM on water. Figure 2(a) shows typical $\Pi-A$ isotherms, suggesting a monolayer-bilayer transition with the plateau indicating coexistence of the two phases [31]. Specular reflectivity $R$, given by the convolution of Eq. (1) with the detector resolution function centered at $\beta=\alpha$, can be used to obtain the structure factor $\Phi_{0}\left(q_{z}\right)$ of the film coated surface. The XR data measured on a PBLG film at points $A=\left(19.2 \AA^{2} /\right.$ monomer, $\left.7.8 \mathrm{dyn} / \mathrm{cm}\right)$ and $B=\left(9.7 \AA^{2} /\right.$ monomer, $\left.8.8 \mathrm{dyn} / \mathrm{cm}\right)$ in the isotherm are shown as $R / R_{F}$ in Fig. 2(b). The solid curves in Fig. 2(b) are fits based on a box model [28] for $\left\langle\rho_{T=0}(z)\right\rangle$. Assuming that the smallest capillary wavelength is on the order of the PBLG rod diameter $d \sim 12.6 \AA$, we take the value for $q_{\max } \sim 2 \pi / d=0.5 \AA^{-1}$. The fact that the results are not sensitive to the precise value of $q_{\max }$ is discussed elsewhere [20,21]. The profiles $\left\langle\rho_{T=0}(z)\right\rangle$ plotted in Fig. 2(c) correspond to a best fit. The film is clearly a monolayer at 

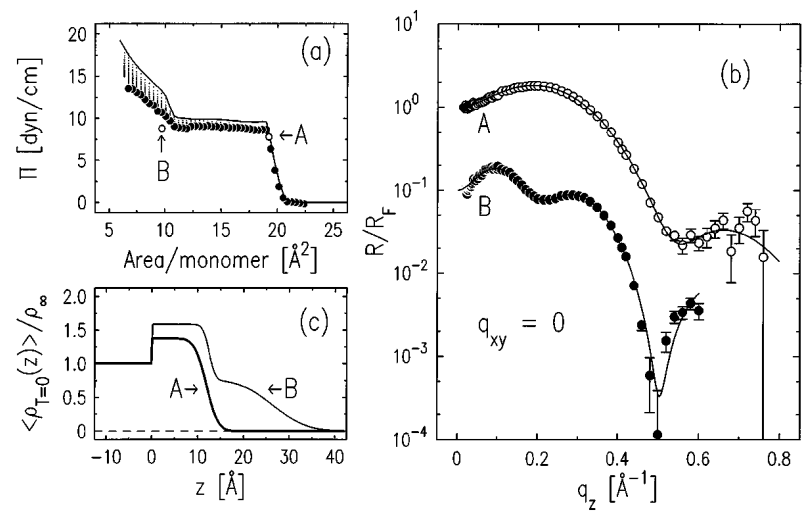

FIG. 2. (a) $\Pi-A$ isotherms for PBLG films at $T=23{ }^{\circ} \mathrm{C}$, showing a continuous scan (-) and a relaxation scan $(-)$, in which the film is relaxed at given $A$ until $\Delta \Pi$ over $5 \mathrm{~min}$ is $<0.05 \mathrm{dyn} / \mathrm{cm}$. (b) Normalized reflectivity $R / R_{F}$ at points $A$ and $B$ in (a). Fits (-) are based on Eq. (1), detector resolutions, and average local electron densities in (c), where $q_{\max }=0.5 \AA^{-1}$ is assumed.

$A$ and an incomplete bilayer at $B$, with each layer thickness being close to the PBLG rod diameter.

Measured differences $\Delta I / I_{0}$ vs $\beta$ from the PBLG film on water are shown in Fig. 3(a) for the monolayer at $A$ and in Fig. 3(b) for the bilayer at $B$. The solid lines correspond to the theoretically predicted $\Delta I / I_{0}$ using Eq. (1), the known parameters $[T, \gamma, \delta \beta, \delta(2 \theta)$, etc.], and the XRbased density profile $\left\langle\rho_{T=0}(z)\right\rangle$. The very good agreement between data and theory shown in Fig. 3(a) justifies the assumption that all of the surface diffuse scattering is TDS from the capillary fluctuations, and that the monolayer is homogeneous. By contrast, Fig. 3(b) shows that the measured scattering from the PBLG bilayer at $B$ exceeds the values predicted on the assumption of a homogeneous bilayer by up to a factor of 2 . In view of the fact that the structure factor $\Phi_{0}\left(q_{z}\right)$ was obtained by fitting the XR, the data near $\beta=\alpha$ agree with the model; however, the off-specular intensity is consistently higher than predicted. This is more clearly evident in Fig. 4, showing the ratio of the data to the solid curves in Fig. 3(b).

In case of lateral density or noncapillary height fluctuations at the interface, $d \sigma / d \Omega$ has another term beyond (1) that corresponds to scattering due to nonzero $\delta \rho_{T=0}(\mathbf{r})=$ $\rho_{T=0}(\mathbf{r})-\left\langle\rho_{T=0}(z)\right\rangle$. Both the lower density in the second layer and the diffuseness of the layer/gas interface evident in $\left\langle\rho_{T=0}(z)\right\rangle$ for the PBLG bilayer [Fig. 2(c)] are suggestive of greater density fluctuations in the newly formed second layer than in the first layer. Therefore, it is reasonable to assume that the extra scattering comes mostly from microscopic inhomogeneities in the second layer. Examples of possible microscopic origins include (i) a distribution in the heights of molecular centers in the second layer, (ii) deviations in the orientation of molecular axes from being parallel to the interface, and (iii) molecular density variations within the second layer.

Since our data cannot distinguish between these, we analyze the excess scattering by modeling the second-

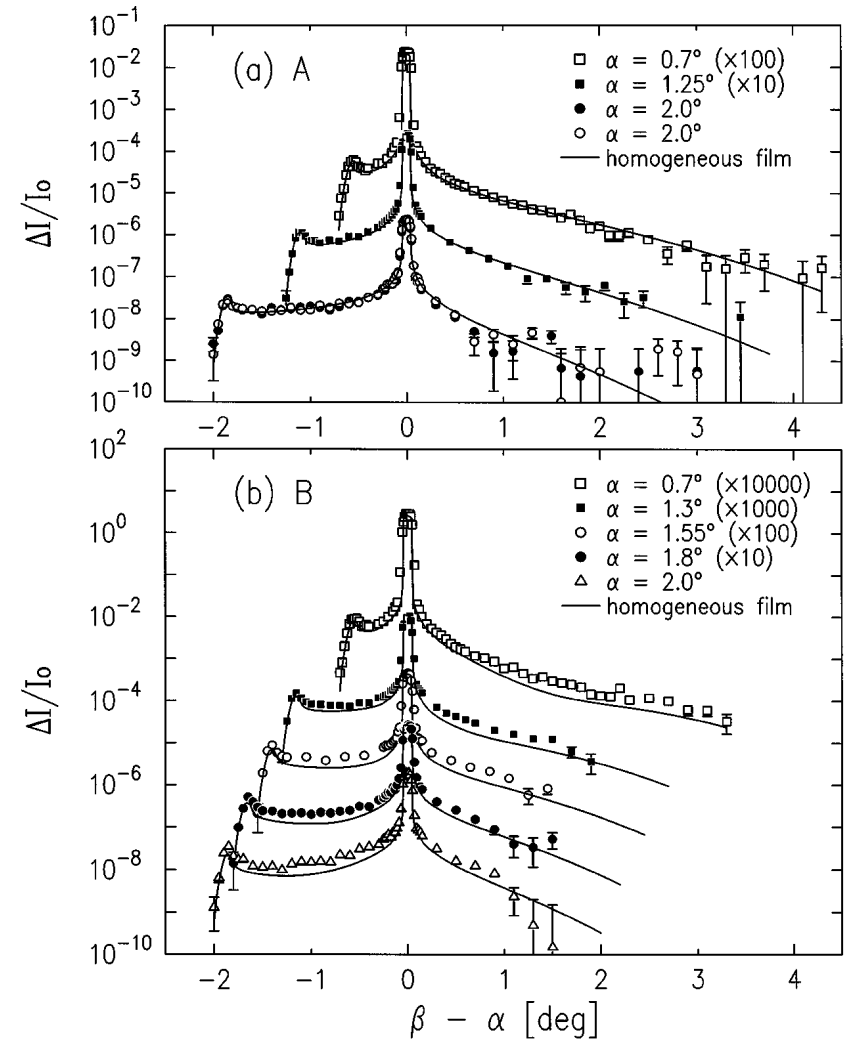

FIG. 3. Measured $\Delta I / I_{0}$ vs $\beta-\alpha$ for PBLG (a) monolayer at $A$ and (b) bilayer at $B$ in Fig. 2(a). The solid curves (-) theoretically expected for homogeneous PBLG films are based on Eq. (1), detector resolutions, and $\left\langle\rho_{T=0}(z)\right\rangle$ in Fig. 2(c).

layer inhomogeneity as follows. The local electron density within the second layer is assumed to be constant at $\rho_{2}=\rho_{\infty} \phi_{2}$, but the height $h_{2}\left(r_{x y}\right)$ of the second layer/gas interface fluctuates about $\left\langle h_{2}(0)\right\rangle=0$ over the surface, where $h_{2}\left(r_{x y}\right)$ is assumed to be laterally isotropic [32] and defined in a frame $\left(\mathbf{r}_{x y}, z^{\prime}\right)$ in which capillary waves are absent [i.e., $z^{\prime}=z-h\left(r_{x y}\right)$ ]. If the two height distributions
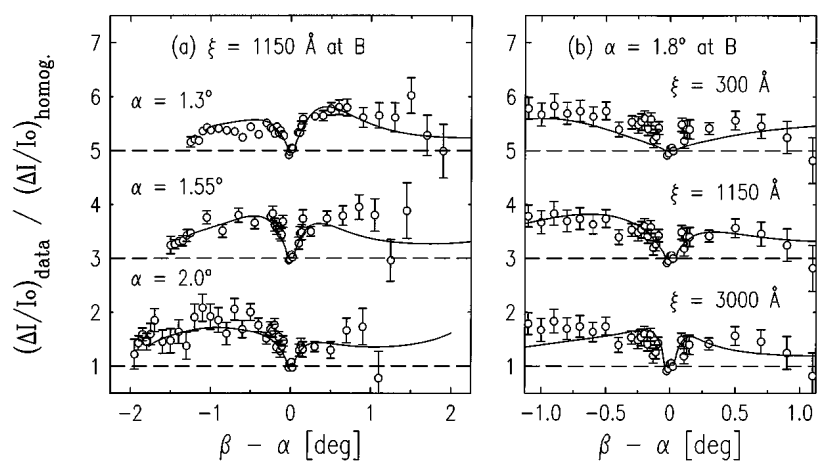

FIG. 4. The ratio of measured $\Delta I / I_{0}$ to the homogeneous contribution in Fig. 3(b) for the PBLG bilayer at $B$. The fits (-) in (a) are based on an inhomogeneous model Eq. (2) with roughness $\sigma_{2}=2.2 \AA$ and correlation length $\xi=1150 \AA$ for the second layer/gas interfacial height fluctuations. The solid curves in (b) correspond to the $\Delta I / I_{0}$ ratio calculated for three different values of $\xi$. 
$\left\{h\left(r_{x y}\right)\right\}$ and $\left\{h_{2}\left(r_{x y}\right)\right\}$ are statistically independent, then, in the limit that $c_{2}\left(r_{x y}\right) \equiv\left\langle h_{2}\left(r_{x y}\right) h_{2}(0)\right\rangle \ll 1 / q_{z}^{2}$, it can be shown that the second term in the total $d \sigma / d \Omega$ is given by the convolution of the capillary $(h)$ and the noncapillary $\left(h_{2}\right)$ fluctuations in reciprocal space,

$$
\begin{aligned}
\frac{1}{A_{0}}\left(\frac{d \sigma}{d \Omega}\right)_{1} \cong & \frac{N \phi_{2}^{2} e^{-\sigma_{2}^{2} q_{z}^{2}}}{2 \pi} \int_{q_{x y}^{\prime}<q_{\max }} d^{2} \mathbf{q}_{x y}^{\prime} \\
& \times \frac{\eta}{q_{x y}^{\prime 2}}\left(\frac{q_{x y}^{\prime}}{q_{\max }}\right)^{\eta} C_{2}\left(\mathbf{q}_{x y}-\mathbf{q}_{x y}^{\prime}\right),
\end{aligned}
$$

where $\sigma_{2}^{2}=\left\langle h_{2}^{2}(0)\right\rangle$ and $C_{2}\left(\mathbf{q}_{x y}\right)$ is the 2D FT of $c_{2}\left(r_{x y}\right)$ [33]. Assuming a simple exponentially decaying correlation function $c_{2}\left(r_{x y}\right)=\sigma_{2}^{2} \exp \left(-r_{x y} / \xi\right)$, so that $C_{2}(q)=$ $2 \pi \sigma_{2}^{2} \xi^{2}\left[1+\xi^{2} q^{2}\right]^{-3 / 2}$, the difference between measured and theoretical $\Delta I / I_{0}$ for a homogeneous PBLG bilayer at various sets of $(\alpha, \beta)$ has been simultaneously fitted to the convolution of (2) with the resolution function. The relative second-layer density was fixed at the XR-based value of $\phi_{2}=0.80$, and only the roughness $\sigma_{2}$ and the correlation length $\xi$ were allowed to vary. The best fit is obtained with $\xi=1150 \AA(400<\xi<3200 \AA)$ and $\sigma_{2}=2.2 \AA$ $\left(1.7<\sigma_{2}<3.1 \AA\right)$. The ratio between the $\Delta I / I_{0}$ calculated from the best fit to the excess scattering and the homogeneous contribution is plotted as the solid lines in Fig. 4(a) at various $\alpha$. The ratio is unity at $\beta=\alpha$ and increases above unity as $\beta$ moves away from $\alpha$. The inverse width of the "valley" centered at $\beta=\alpha$ is a measure of correlation length $\xi$. This is demonstrated in Fig. 4(b), in which the ratios at three different values of $\xi$ are plotted at $\alpha=1.8^{\circ}$. From the above analysis, we estimate the correlation length associated with the second-layer inhomogeneity to be on the order of $\xi \sim 1000 \AA$, which is about 80 times the rod diameter or about 7-8 times the rod length of typical PBLG molecules.

In summary, we have proposed a sum rule that establishes the absolute magnitude of the capillary wave contribution to the differential cross section (1) for low-angle $\mathrm{x}$-ray scattering from liquid surfaces. Validity of the sum rule is demonstrated by the excellent agreement between measurements on a bare water surface $\left[\Phi_{0}\left(q_{z}\right)=1\right]$ and a PBLG monolayer $\left[\Phi_{0}\left(q_{z}\right) \neq 1\right]$ and intensities $\Delta I / I_{0}$ calculated by numerical convolution of (1) with the slitdefined resolution function. Analysis of excess diffuse scattering from a PBLG film compressed beyond the elastic limit of the monolayer established the possibility for quantitative characterization of noncapillary inhomogeneities on liquid surfaces. On the basis of the analysis leading to Fig. 4 surface fluctuations with 2D correlation lengths between $\sim 100 \AA$ and $1 \mu \mathrm{m}$ can readily be studied.

The Harvard contribution to this work was supported by Grants No. NSF-DMR-95-23440 and No. NSF-DMR94-00396. Work at the University of Massachusetts was supported by Grant No. NSF-DMR-95-10031. Beamline
X22B at NSLS, Brookhaven National Laboratory is supported by No. DE-AC02-76CH00016.

[1] H.L. Swinney and D. L. Henry, Phys. Rev. A 8, 2586 (1973).

[2] Light Scattering Near Phase Transitions, edited by H.Z. Cummins and A.P. Levanyuk (North-Holland, Amsterdam, 1983).

[3] H. Chen, R. J. Comstock, and J. B. Cohen, Annu. Rev. Mater. Sci. 9, 51 (1979).

[4] P.S. Pershan, Structure of Liquid Crystal Phases (World Scientific, Singapore, 1988).

[5] H. Dosch, Critical Phenomena at Surfaces and Interfaces: Evanescent $X$-ray and Neutron Scattering (SpringerVerlag, Berlin, 1992).

[6] H. Dosch, Appl. Phys. A 61, 475 (1995).

[7] S. Dietrich and H. Wagner, Z. Phys. B 56, 207 (1984).

[8] D. R. Nelson, in Phase Transitions and Critical Phenomena, edited by C. Domb and J. L. Lebowitz (Academic, New York, 1983), Vol. 7, p. 1.

[9] D. R. Nelson and B.I. Halperin, Phys. Rev. B 19, 2457 (1979).

[10] S. K. Sinha et al., Phys. Rev. B 38, 2297 (1988).

[11] S. Dietrich and A. Haase, Phys. Rep. 260, 1 (1995).

[12] J. Daillant et al., J. Chem. Soc. Faraday Trans. 92, 505 (1996).

[13] D. K. Schwartz et al., Phys. Rev. A 41, 5687 (1990).

[14] M. K. Sanyal et al., Phys. Rev. Lett. 66, 628 (1991).

[15] W. Zhao et al., J. Chem. Phys. 97, 8536 (1992).

[16] L. Bourdieu et al., Phys. Rev. Lett. 72, 1502 (1994).

[17] Z. Li et al., Langmuir 11, 4785 (1995).

[18] P. Fontaine et al., J. Phys. II (France) 7, 401 (1997).

[19] C. Gourier et al., Phys. Rev. Lett. 78, 3157 (1997).

[20] H. Tostmann et al. (to be published).

[21] M.P. Gelfand and M.E. Fisher, Physica (Amsterdam) 166A, 1 (1990).

[22] A. Braslau et al., Phys. Rev. A 38, 2457 (1988).

[23] J. Daillant et al., J. Phys. II (France) 1, 149 (1991).

[24] Li et al. (Ref. [17]) have previously used a scattering geometry similar to OSDX to separate the capillarywave TDS from small-angle diffraction originating from interparticle correlations of micelles at the air-water interface.

[25] In order to account for surface enhancement effects when $\alpha, \beta \sim \alpha_{c}$, transmission factors for the single watervapor interface are included in Eq. (1). The effect of multiple interfaces is negligible here.

[26] Sinha et al. (Refs. [10,14]) obtained similar results analytically for one model resolution function.

[27] D. K. Schwartz, M. L. Schlossman, and P.S. Pershan, J. Chem. Phys. 96, 2356 (1992).

[28] M. Fukuto et al., J. Chem. Phys. 107, 5531 (1997).

[29] Sigma Chem. Co. MW: 26,000 (vis), 17,300 (LALLS).

[30] S. M. Yu et al., Nature (London) 389, 167 (1997).

[31] P. Lavigne et al., Thin Solid Films 242, 229 (1994).

[32] The range of $\beta-\alpha$ is not sufficient to probe the rodinduced anisotropy in the pair correlations.

[33] Equivalent results can be obtained in terms of lateral density-density correlations. 